\title{
Article
}

\section{Experiences of Improving Access to Psychological Therapy Services for Perinatal Mental Health Difficulties: a Qualitative Study of Women's and Therapists' Views}

Millett, Lottie, Taylor, Billie Lever, Howard, Louise M, Bick, Debra, Stanley, Nicky and Johnson, Sonia

Available at http://clok.uclan.ac.uk/20665/

Millett, Lottie, Taylor, Billie Lever, Howard, Louise M, Bick, Debra, Stanley, Nicky ORCID: 0000-0002-7644-1625 and Johnson, Sonia (2017) Experiences of Improving Access to Psychological Therapy Services for Perinatal Mental Health Difficulties: a Qualitative Study of Women's and Therapists' Views. Behavioural and Cognitive Psychotherapy . pp. 1-16. ISSN 1352-4658

It is advisable to refer to the publisher's version if you intend to cite from the work. http://dx.doi.org/10.1017/S1352465817000650

For more information about UCLan's research in this area go to http://www.uclan.ac.uk/researchgroups/ and search for <name of research Group>.

For information about Research generally at UCLan please go to http://www.uclan.ac.uk/research/

All outputs in CLoK are protected by Intellectual Property Rights law, including Copyright law. Copyright, IPR and Moral Rights for the works on this site are retained by the individual authors and/or other copyright owners. Terms and conditions for use of this material are defined in the policies page. 


\title{
Experiences of Improving Access to Psychological Therapy Services for Perinatal Mental Health Difficulties: a Qualitative Study of Women's and Therapists' Views
}

\author{
Lottie Millett \\ Division of Psychiatry, University College London, UK \\ Billie Lever Taylor \\ Division of Psychiatry, University College London, UK
}

Louise M. Howard

Section of Women's Mental Health, Institute of Psychiatry, Psychology and Neuroscience, King's College London, UK

Debra Bick

Florence Nightingale Faculty of Nursing and Midwifery/Women's Health Division, King's College London, UK

Nicky Stanley

School of Social Work, Care and Community, University of Central Lancashire, UK

Sonia Johnson

Division of Psychiatry, University College London, UK

Background: Perinatal mental health difficulties are highly prevalent. In England, the Improving Access to Psychological Therapy (IAPT) programme provides evidence-based psychological treatment, predominantly in the form of brief manualized cognitive behavioural

Correspondence to Billie Lever Taylor, Division of Psychiatry, University College London, Maple House, 149 Tottenham Court Road, London W1T 7NF, UK. E-mail: billie.taylor@ucl.ac.uk 
therapy (CBT), to people with mild to moderate depression or anxiety. Yet little is known about the experiences of women referred to IAPT with perinatal mental health difficulties. Aims: The aim of this qualitative study was to investigate how women view IAPT support for perinatal mental health. We also gained the perspective of IAPT therapists. Method: Semi-structured interviews were conducted with twelve women who had been referred to and/or received therapy from IAPT during the perinatal period. Additionally, fourteen IAPT therapists participated in two focus groups. Thematic analysis was used. Results: Key themes centred on barriers to access and the need to tailor support to (expectant) mothers. Women and therapists suggested that experiences could be improved by supporting healthcare professionals to provide women with more help with referrals, better tailoring support to the perinatal context, improving perinatal-specific training, supervision and resources, and offering a more individualized treatment environment. Conclusions: Overall, women reported positive experiences of support offered by IAPT for perinatal mental health difficulties. However, services should seek to facilitate access to support and to enable therapists to better tailor treatment.

Key words: IAPT, perinatal mental health, CBT, qualitative methods

\section{Introduction}

Perinatal mental health difficulties are very common (Howard et al., 2014), with an estimated $15 \%$ of women experiencing depression or anxiety in pregnancy or the first year post-birth (Khan, 2015). These difficulties are associated with a range of negative consequences. For example, there is a large body of evidence suggesting a relationship between perinatal mental health difficulties and possible adverse consequences for the child, particularly in women with chronic difficulties (Stein et al., 2014), making prompt, effective treatment particularly important in pregnancy and after childbirth.

From a cost perspective, there is a large financial burden. In the USA, women with postpartum depression have been found to incur $90 \%$ higher health services expenditure than non-depressed postnatal women (Dagher et al., 2012). In the UK, it is estimated that the cost to society of perinatal depression, anxiety and psychosis reaches $£ 8.1$ billion every year (Bauer et al., 2014), partly as a result of ineffective or absent treatment, resulting in longer and more severe episodes (Gavin et al., 2015).

Internationally, many clinical guidelines recommend a 'stepped care' approach to treatment for mental health difficulties (Richards et al., 2010), where simpler first-line treatments are offered initially and only those who do not (or are unlikely to) benefit are offered more intensive support. In England, the Improving Access to Psychological Therapy (IAPT) programme was introduced in 2007 within the National Health Service (NHS), with the aim of increasing the availability of routine evidence-based therapies, predominantly based on cognitive behavioural therapy (CBT) principles, for mild to moderate depression and anxiety (Clark, 2011). IAPT follows a stepped care approach, where 'low intensity' treatments, consisting of brief manualized therapy, such as guided self-help and computerized therapy, are delivered by graduates with mental health training known as psychological wellbeing practitioners (PWPs). 'High intensity' therapy, delivered by more experienced therapists, is reserved for people with more complex difficulties or who do not respond to low intensity treatments. Data on the number of women accessing IAPT for perinatal mental health difficulties are not routinely collected, but one pathfinder site estimated that they accounted for 5-11\% of all IAPT service users (Department of Health, 2013), emphasizing the importance of catering adequately for this population. 
Overall, service users report satisfaction with IAPT, although some say it is insufficiently individualized or too brief (Hamilton et al., 2011). Of particular importance, there is evidence that CBT may be less effective for perinatal difficulties than other disorders (Cuijpers et al., 2008,2010 ), although it is acknowledged that this may partly reflect limitations of current trials in this field. Nevertheless, it has been suggested that outcomes and adherence may be improved by better tailoring interventions to perinatal women (O'Mahen et al., 2012). Tailoring can include offering greater flexibility, for example with the timing and location of appointments, as well as tailoring the content of therapy itself. Indeed, research has suggested that manualized behavioural interventions, specifically adapted to the perinatal context, and offered with support from IAPT therapists, can be effective for women with post-partum depression, with results suggesting that women engage better with a more tailored treatment (O'Mahen et al., 2014).

In line with this, it has been argued that IAPT services should tailor support to the perinatal context, for example by offering therapists additional training, permitting babies in sessions and offering longer sessions to accommodate the need to feed/change the baby (Department of Health, 2013). A perinatal mental health care skills competency framework is currently being piloted with healthcare professionals in the UK (Health Education England, 2017). However, at the time of this study there were no perinatal-specific interventions or resources within IAPT, and concerns have been raised that training schemes for IAPT therapists place insufficient emphasis on perinatal mental health (Joint Commissioning Panel for Mental Health, 2012).

Nevertheless, little is known about how women or IAPT therapists themselves view IAPT for perinatal mental health difficulties. One small study within an IAPT service found that women who accessed CBT for postnatal depression reported a lack of information about postnatal depression among healthcare professionals and reported difficulty accessing support via their family doctors (known as general practitioners [GPs] in the UK). They valued choice in the mode of therapy (e.g. group, individual or telephone), and felt that crèche facilities and home visits were important (S. Hovington, unpublished presentation from 2012: Postnatal depression: barriers to engagement and retention in a primary care psychological service). However, this study consisted of just one focus group with six women from a single IAPT service and therefore its findings are only tentative. The current study aimed to further inform treatment by exploring women's and therapists' experiences of IAPT for perinatal mental health difficulties.

\section{Method}

This study was nested within a larger qualitative study investigating stakeholders' experiences of perinatal mental health care, itself part of a wider mixed-method programme exploring the effectiveness of services for mothers with mental illness. A qualitative research design was used to allow in-depth analysis and exploration of themes that may have been unanticipated.

\section{Setting}

The study took place in IAPT services across England, with the majority in London. Interviews took place face-to-face in participants' homes or neutral locations. Focus groups took place within healthcare settings. NHS ethical approval was obtained and all participants provided informed consent. 


\section{Participants}

Service users. Twelve women were recruited who had used or been referred to IAPT, a number considered sufficient for data saturation (Guest et al., 2006). Eligible women were initially identified and approached by a (IAPT) clinician. Those expressing an interest were contacted by a researcher. Nobody dropped out after being contacted, although one additional potential participant was not contactable. It is not possible to quantify how many women refused to participate as clinicians only informed the researchers of those interested. In some cases participants were recruited through non-IAPT services but reported input from IAPT during the interview. The IAPT-relevant information from these interviews was used.

Therapists. Two focus groups were carried out with a total of fourteen IAPT therapists. Therapists were recruited via poster advertisements and contacts working with IAPT. One focus group consisted of twelve London-based IAPT therapists who were perinatal leads for their teams. The second was a mixed group of eight practitioners from a range of services across a Northern NHS health provider site. This group included two IAPT therapists whose comments were included.

\section{Inclusion criteria}

Service users:

- English-language speaker

- Woman aged 16 or over

- Referral to/use of IAPT for a perinatal mental health difficulty during pregnancy or post-birth

- Baby aged 6-9 months at the time of interview

Therapists:

- Clinician within an IAPT service

\section{Data collection}

Service users. Interviews were carried out between September 2015 and November 2016. An interview guide was developed by the research team and amended following review by a service user and carer advisory panel, along with piloting with five women who had accessed perinatal mental health support. The guide covered topics including experiences of accessing and receiving support, involvement of women's families, information and choice. Demographic data were also recorded.

Semi-structured interviews, lasting approximately one hour, were primarily conducted by the second author (a mother, clinical psychologist and researcher specializing in perinatal mental health). Others were conducted by research assistants and the first author (a master's student within a London University's Psychiatry department, who had herself accessed IAPT and had experience delivering manualized CBT interventions). Qualitative research acknowledges that researchers inevitably influence how data are collected and analysed. Here, the researchers' personal experiences of motherhood and of working in and/or accessing perinatal/IAPT services may have influenced their expectations, assumptions and interpretations of the data. 
Therapists. Focus groups with therapists lasting approximately 1.5 hours were run by the second author in November and December 2016. Therapists were shown a short presentation of emerging findings from the interviews with women collected for this study, and asked to discuss these and their own experiences of supporting women with perinatal mental health difficulties.

\section{Analysis}

Interviews and focus groups were audio-recorded, transcribed verbatim, anonymized and imported to QSR NVivo 11. Data were coded by the first author using thematic analysis, a rigorous qualitative method that allows inductive analysis of rich complex data (Braun and Clarke, 2006). Line-by-line coding generated initial codes. Relationships between codes were then explored and codes were combined into themes using an iterative process to develop a comprehensive, nuanced understanding of the data. Validity was increased by the second author separately coding six interviews and both focus groups. Any disagreements were discussed and a consensus reached.

\section{Results \\ Demographics}

Service users. The women's demographics are shown in Table 1. Two-fifths of women ( $n$ $=5$ ) were White British. The mean age was 34 years (age range $21-43$ years). Few data are available to compare these figures to IAPT service users overall or, more specifically, to women accessing IAPT for perinatal mental health difficulties. However, the majority of IAPT service users are 18-35 years old, while the overall proportion from a White British background is $84 \%$ (Health and Social Care Information Centre, 2014).

Six women were 6 months postnatal at the time of the interview, three were 8 months postnatal, and three were 9 months postnatal. Ten of the twelve participants had received treatment from IAPT, while two had been referred but not yet taken up treatment. These participants were included to give greater insight into barriers to accessing support. Nine of the ten participants who had received treatment said this was individual face-to-face therapy, while one received online therapy. Seven received treatment postnatally, one antenatally, and two both antenatally and postnatally. All ten described receiving 'CBT' although it was not always clear what exactly this consisted of (e.g. low versus high intensity) or by whom it was delivered.

Therapists. Table 2 shows therapists' demographics. All therapists were female. Five described themselves as 'psychological wellbeing practitioners' (PWPs), four as 'cognitive behavioural therapists', one as a 'psychological therapist', two as 'clinical psychologists', and two as 'deputy leads'.

\section{Thematic analysis}

Overall, women reported positive experiences of receiving psychological support from IAPT. However, women and therapists alike highlighted issues relating to barriers to access and a lack of adaptation to the perinatal context. 
Table 1. Demographics of the service users

\begin{tabular}{|c|c|}
\hline Participant characteristics & $n$ \\
\hline \multicolumn{2}{|l|}{ Ethnicity } \\
\hline White British & 5 \\
\hline White other & 3 \\
\hline Black other & 1 \\
\hline Black Caribbean & 1 \\
\hline Arab & 1 \\
\hline Asian & 1 \\
\hline \multicolumn{2}{|l|}{ Location } \\
\hline London & 9 \\
\hline South East of England & 3 \\
\hline \multicolumn{2}{|l|}{ Age (years) } \\
\hline $16-21$ & 1 \\
\hline $22-27$ & 0 \\
\hline $28-33$ & 3 \\
\hline $34-39$ & 6 \\
\hline $40-45$ & 2 \\
\hline \multicolumn{2}{|l|}{ Diagnosis } \\
\hline Depression & 6 \\
\hline Anxiety & 4 \\
\hline Depression and anxiety & 2 \\
\hline \multicolumn{2}{|l|}{ Highest education level } \\
\hline No formal qualifications & 2 \\
\hline GCSEs & 1 \\
\hline NVQ & 1 \\
\hline A-levels & 1 \\
\hline Bachelor's degree & 4 \\
\hline Master's Degree & 3 \\
\hline \multicolumn{2}{|l|}{ Marital status } \\
\hline Married/cohabiting & 8 \\
\hline Single/separated & 4 \\
\hline \multicolumn{2}{|l|}{ Number of months postnatal } \\
\hline 6 & 6 \\
\hline 8 & 3 \\
\hline 9 & 3 \\
\hline \multicolumn{2}{|l|}{ Number of children } \\
\hline 1 & 6 \\
\hline 2 & 2 \\
\hline 3 & 2 \\
\hline $4+$ & 2 \\
\hline
\end{tabular}

Barriers to access

Service user findings. Women were in contact with a range of professionals during and after their pregnancies, including midwives, health visitors (community public health nurses) and GPs. They relied on these professionals to help them identify that they had a mental health 
Table 2. Demographics of the therapists

\begin{tabular}{ll}
\hline Participant characteristics & $n$ \\
\hline Ethnicity & \\
White British & 6 \\
White Irish & 2 \\
Asian & 4 \\
Black African & 1 \\
Mixed White and Black African & 1 \\
Location & \\
London & 12 \\
North of England & 2 \\
Job title/role & 5 \\
Psychological wellbeing practitioner (PWP) & 4 \\
Cognitive behavioural therapist & 2 \\
Clinical psychologist & 2 \\
Deputy lead & 1 \\
Psychological therapist & 12 \\
Perinatal lead & \\
Number of years in role & 1 \\
Up to 1 & 7 \\
$1-2$ & 3 \\
$3-5$ & 3 \\
$6+$ & \\
Number of years working with women with perinatal mental health difficulties & 6 \\
$1-2$ & 4 \\
$3-5$ & 1 \\
$6-9$ & 3 \\
$10+$ & \\
\hline
\end{tabular}

difficulty and refer them to IAPT. Sometimes this process worked well, particularly where there was continuity and where professionals appeared well-informed about perinatal mental health and available support. For example, one woman reported that her midwife:

'... did everything she should have done. Which is identify the problem, get me to the right person, provide kind of interim just talking counselling and then, once I could get to someone, then she could step away ... I'm lucky I had her because she knew how to work through the system a bit.' [Mother 4]

However, ten of the twelve women reported some issues with accessing IAPT. In some cases, women found it difficult to identify that they had a mental health difficulty, and felt professionals did not help them understand how this might present.

'No one speaks to you about postnatal depression. Like when you're pregnant nobody speaks to you about it ... When I went for my six week [postnatal] check [my GP] did ask me "Do you have any postnatal depression?" They just expect you to know...It's not like, "Do you feel low?" or "Do you feel certain things?", [Mother 12] 
Women were also unsure whether it was relevant to talk about their mental health with midwives and health visitors.

'[The health visitor] didn't ask, but I didn't feel the need to say anything, because I just thought that's just a personal issue so why would I tell them about what happened?' [Mother 12]

Indeed, five of the twelve women felt the stigma of seeking help made it difficult to access support due to:

'Feeling that you should cope... so I shouldn't need to ask for help.' [Mother 9]

Women feared losing custody of their baby if professionals felt:

'Oh, you can't cope? We'll take the baby then.' [Mother 9]

When women did disclose difficulties, at times they felt the focus was on medication rather than therapy, and there was confusion around referring them to IAPT.

' $[$ My GP] was very quick to prescribe medication ... and then there was a whole conversation with her about what else was available and there was a real confusion ... So this was like, when you're kind of in the throes of it, the last confusion that you need really.' [Mother 2]

Where they were given contact details for IAPT, women sometimes reported reluctance to contact services independently.

'It's fine to post a number on the back of a flyer, but who calls those numbers?' [Mother 4]

Misunderstandings or insufficient explanations of IAPT could also prevent access. For example, one woman (Mother 5) believed the number given to self-refer to IAPT was a crisis line, while another (Mother 8) believed only group therapy would be offered.

Although there were issues with access, once women were referred to IAPT, they were often prioritized and assessed within 2 weeks, although some felt the wait to treatment postassessment was too long.

\section{Therapist findings}

IAPT therapists similarly emphasized the importance of GPs, midwives and health visitors helping refer women to IAPT. They felt women were frequently left to self-refer, but struggled to do so:

'GPs will say self-refer. And we have brought this up with GPs that if people are in distress they're unlikely to refer... And I think they need to take some responsibility of actually doing the referral with them or, with their consent for them, including midwives and children's centres. I think there's such reliance now on self-referral.' [Deputy Lead]

Therapists reported that available literature about IAPT often: 'doesn't say anything ... about baby problems' [PWP] and midwives and health visitors sometimes: 'don't even know what IAPT means' [CBT therapist] or don't like asking women about their mental health: 'because 
they actually don't know what to do with the information. Just do not know. So like, we don't ask. Or they'll just say "you're not feeling depressed, are you?" , [Clinical Psychologist]

Therapists emphasized the importance of providing practitioners with training about mental health and IAPT and establishing close links: 'If the people on the ground, midwives and the health visitors, are not being told anything about IAPT, then how can they facilitate?' [CBT therapist]

The need to tailor support to (expectant) mothers

Service user findings

Overall, women found IAPT helpful, with seven of the ten women who had accessed therapy reporting generally positive experiences. Women valued the way CBT helped them 'separate fact from fiction' [Mother 3] and 'made me see what triggered the fear.' [Mother 1] They also valued having a normalizing, non-judgemental therapist who enabled them to 'just say anything... even things that I would never say to...other mums.' [Mother 11]

However, whilst IAPT was valued overall, eight of the ten women who had accessed IAPT felt it could be better tailored to the perinatal context. Where more tailored support was offered, women valued this:

'It was absolutely tailored because [my therapist] has a lot of specific experience working with women postnatal... She did a really nice job. ' [Mother 4]

Further, one woman felt online support was well-tailored to her perinatal context because 'If the baby starts screaming then I can just press stop and then get back to it.' [Mother 2]

Where tailoring was absent, women wished support had been adapted: 'I've never experienced anxiety before, the first time I'm experiencing it is after being a mum. So I believe it's probably to do with the postnatal [sic]. If it was more catered more to that I think it'd be really helpful.' [Mother 12]

\section{Content of sessions and homework}

More specifically, women felt that IAPT materials could be improved by including examples with women and babies, and said 'homework' tasks were at times unrealistic: 'It was really helpful but she gave me a lot of homework and realistically I was feeding them around the clock and I just didn't do it.' [Mother 3]

Women also reported that IAPT treatment was very problem-specific, with little remit for discussing related issues that might also be prominent in the perinatal context such as identity and relationship changes:

'It's much more focused on one specific issue. So she puts it in a box and then kind of all the other stuff is off the table, like you don't talk about that ... But there's kind of a lot more around it too which would have been helpful to talk through as well.' [Mother 4]

\section{A non-baby-friendly environment}

Most women said they were not able to bring their baby to sessions or, if they were, it was only a one-off. This resulted in difficulties finding childcare, for example: 'It's hard trying to leave him with people.' [Mother 7] 
Where women were able to take their baby, the environment was not always baby-friendly: 'There is something in the room for little toddlers, like an abacus and stuff like that. But no, he wasn't really, just had his car-seat' [Mother 9] and 'It'd be great if it came with a crèche' [Mother 11]. Although some women worried their babies would be distracting, others nevertheless wanted to bring them:

'I wish that ... I could just take him and if he makes a fuss for 15 minutes when he is doing that then

I lose the 15 minutes ... When I asked "Can I bring my baby?" ... "It's not recommended" [my therapist] said.' [Mother 6]

There was one report of a positive experience of bringing a baby where, although the environment wasn't baby-friendly, the therapist was considerate of the mother's and baby's needs:

'She did a nice job of if I needed to stay and feed the baby, like breastfeed the baby, she could leave me in the room afterwards. And so that was just really nice that she was thoughtful about it.' [Mother 4]

\section{Involving fathers}

It was notable that fathers/partners were not involved in any of the women's therapy, yet eight of the ten women who accessed IAPT wanted the father to be involved, in some way: 'so he can understand what I'm going through' [Mother 4] and so 'he could give his viewpoint'. [Mother 12]

Whilst most women had not questioned this, one did ask for the father to be involved but was told it was not possible:

'I did ask if I could take my husband with me... to the therapy, yes, but they said no. So I was really nervous. My husband came with me, but he had to sit outside.' [Mother 12]

\section{Flexibility, individualization and choice}

Overall, women felt that therapists were flexible with appointments, with half saying they were offered more sessions, or therapists were considerate of last-minute cancellations given they had a baby:

'She has said all along, like, if you need more, you always can. And she's been really understanding if I haven't made it because I'm knackered and forgotten.' [Mother 9]

However, women's experiences also seemed to be marked by feeling they had a lack of choice and that IAPT could feel process-driven and impersonal. The IAPT assessment was one example where women were not given a choice even though some found a telephone assessment uncomfortable:

'It's more difficult to like someone over the phone than when you see someone ... It did not feel as comfortable. I don't know, over the phone I feel more evaluated.' [Mother 6]

The focus on outcome measures and form-filling was also disliked by some: 
'I actually thought ... they're going to sit down and just listen to my problems ... But it wasn't like that... Just like filling in that form, just telling you how you're feeling, and, like, it wasn't like, "why are you feeling like that?" ' [Mother 7]

Overall, women said they were: 'not given choices, it was like here's what we could offer you, do you want it?' [Mother 3] and that: 'It's a bit of a production line of, get this person in for their six [sessions]. That's what it feels like.' [Mother 10]

\section{Therapist findings}

Therapists similarly highlighted difficulties tailoring IAPT to the perinatal context. Overall, they described IAPT as 'too rigid' [CBT therapist] and felt: 'We're trying to do our best for mum and baby and partner, but it's just so difficult in an IAPT setting. ' [Deputy Lead]

\section{Constraints of the IAPT framework}

While therapists said they tried to be flexible, for example offering mothers: ' $a$ few more sessions' [PWP], this was not always easy:

'Sometimes it has been so difficult where they've breached the policy for like cancelling notice and these kind of things.' [CBT therapist]

'Unfortunately there are constraints, whether it be kind of structural in terms of the buildings that we work in, or the ... rather relentless IAPT targets and the model.' [Clinical Psychologist]

Some therapists were also concerned that being too flexible could undermine the effectiveness of treatment: 'There is a tension between wanting to be nice and helpful with what's actually going to work.' [Clinical Psychologist]

\section{Lack of perinatal-specific training and resources}

Therapists felt that to work effectively within the perinatal context: 'You need to be assertive clinicians to advocate for your service users' [CBT therapist]. But some felt deskilled and anxious. For example, one noted: 'I have to adhere to policies. I feel I am more nervous than the actual mother' [CBT therapist], while another argued that: 'Therapists often just feel that they haven't got the confidence to work in that area because they see perinatal as something very specialist.' [CBT therapist]

Therapists spoke about a lack of perinatal training within IAPT, particularly for PWPs, meaning they: 'may not have any clue as to how they tailor.' [Deputy Lead] Therapists suggested setting up specialist perinatal teams within IAPT or having specific training and guidelines for applying IAPT to the perinatal context:

'Nothing actually translates nicely into the IAPT model. So I think that to me certainly feels like that's a real gap ... There doesn't seem to be any really helpful guidelines for IAPT work in perinatal period.' [CBT therapist]

Good supervision was seen as important, but was not always easy to obtain: 'It's not that my supervisor isn't good but she's not, her area isn't perinatal.' [CBT therapist] 
Overall, therapists emphasized that IAPT could be effective for women with perinatal mental health difficulties, but more guidance was needed for delivering treatment to this population: 'It needs to be fleshed out so that it can then be translated into this more routine PWP kind of way.' [CBT therapist]

\section{A non-baby-friendly environment}

While some therapists allowed babies in sessions, others said: 'The general consensus is you just don't have children in the sessions.' [CBT therapist] One therapist reported that: 'I've had to allow some mums to bring their baby in the pram. But when I spoke to my supervisor I was told that we're not allowed to do that.' [PWP]

Some therapists emphasized the importance of allowing babies in sessions, noting that: 'Some of our mums would never get support otherwise because they wouldn't have anyone to look after their children' [PWP] and: 'It seems very important to see the mother with the baby because of what you can see and what you can observe.' [Clinical Psychologist]

However, others had concerns including:

- worries about a mother's distress affecting her baby:

'If you're doing some sort of therapy, perhaps trauma work, I don't think it would be appropriate to have a baby in the session because the mum's going to get so upset.' [CBT therapist]

- concerns about health and safety:

'We had to undergo massive risk assessment and childproof edges and wires ... Make downstairs access, and where's this pram going to go, and how many prams can we fit in at one time.' [Clinical Psychologist]

- and worries that babies can 'be a bit of a distraction.' [PWP]

Some therapists cited positive experiences of using crèches, although others felt time could be lost where babies took time to settle. Nevertheless, therapists felt greater use of crèches would be valuable.

\section{Involving fathers}

Finally, while some therapists had invited fathers to sessions, others said this was not commonplace. Some had set up groups for fathers, but experienced difficulty getting them to attend: 'We ... tried to get fathers in, but the fathers wouldn't come for six weeks, which is why we've basically condensed it into a three hour workshop.' [Clinical Psychologist] They attributed this to stigma, work commitments, and the fact perinatal services tend to be 'femaledominated'.

\section{Discussion}

\section{Main findings}

Overall, women reported positive experiences of receiving support from IAPT for perinatal mental health difficulties. Nevertheless, issues were highlighted by women and therapists relating to barriers to access and a need to better tailor therapy to the perinatal context. 
Barriers to access included a reluctance to disclose difficulties due to stigma and fear of custody loss, as well as lack of clear information and support with referrals from healthcare professionals such as GPs, midwives and health visitors. These issues are similar to those identified in Hovington's (2012) study of IAPT support for postnatal depression, and in wider research exploring women's experiences of accessing care for perinatal mental health difficulties (Megnin-Viggars et al., 2015). Nevertheless, while some women did express dissatisfaction with the wait for treatment, once referred to IAPT women were often prioritized and assessed quickly.

Despite recommendations for support to be tailored to the perinatal context (Department of Health, 2013) this was generally seen as insufficient. For example, the content of sessions was not always perinatal-focused, homework expectations could be unrealistic, there was little opportunity to explore associated issues such as relationship changes, fathers/partners were typically not involved, and babies were seldom welcome, hindering attendance. Women also felt that care could be process-driven and impersonal. Where it was offered, a tailored approach and flexibility were valued. These findings may lend support to O'Mahen's (2012) assertion that differences in effectiveness between CBT for postnatal and non-postnatal populations may be due to a failure to tailor interventions to the perinatal period.

Therapists similarly expressed frustration that the constraints of IAPT prevented them from adapting treatment sufficiently. Yet some also worried that deviating from protocols, or allowing babies in sessions, could reduce treatment efficacy or create other risks. Future research should therefore build on the work of O'Mahen et al. (2014) and directly compare the effectiveness of a tailored perinatal intervention to its generic alternative. Overall, therapists expressed anxiety working with perinatal mental health difficulties and wanted perinatal-specific training, supervision and resources.

\section{Limitations}

This is the first published study to explore experiences of IAPT for perinatal mental health. Nevertheless, it is important to acknowledge the study's limitations. Firstly, the sample was relatively small and included two participants who had not yet accessed IAPT services, as well as a high proportion of London-based women and therapists. Although qualitative research does not aim to be generalizable, it would have been valuable to have a larger, more diverse sample. It may be that this predominantly London-based sample has conveyed a biased picture, although the accounts given suggested a variety of experiences.

As the interviews with women were taken from a larger study exploring experiences of a wide range of services for perinatal mental health difficulties, greater depth might have been gained by conducting separate interviews focused only on IAPT. However, women were probed about each service they reported using, resulting in rich IAPT-specific data.

The therapists included may have been particularly well-informed and interested in perinatal mental health, especially as the majority were perinatal leads for their teams. Their views may therefore have differed from those of other therapists. Furthermore, while the presentation of emerging themes delivered to the therapists prior to the focus groups helped promote rich and pertinent discussion, it is possible that it could also have somewhat primed the therapists' responses.

It is also possible that clinicians may have referred women to the study who they felt would be particularly positive about IAPT. However, many women did report negative aspects to their 
experiences, and some were referred by other services but had also accessed IAPT, helping reduce this potential bias.

Finally, it would be valuable to further explore barriers to access among women who do not ultimately receive support. Although difficulties with access were reported, all but two of the women who took part had successfully accessed support. This sample could therefore represent a particularly motivated or perseverant group of women.

\section{Clinical recommendations}

There appears to be a need for better information for women about perinatal mental health difficulties, and for maternity services and generic mental health services like IAPT to establish closer links with GPs, health visitors and midwives. These practitioners should be provided with relevant training and information and encouraged to support women with referrals.

While caution must be taken to maintain treatment efficacy, the development of specifically tailored perinatal interventions and resources may help generic mental health services better meet the needs of perinatal women. There also seems to be a need for greater accommodation of babies in sessions. There is a paucity of evidence on the impact of this but, to our knowledge, no evidence to suggest babies should be excluded. Improved training for therapists about how to adapt manualized therapy to the perinatal context would appear essential to increase confidence and skills. Keeping accurate records in IAPT of perinatal cases, the type of therapy delivered and any adaptations made would help future robust quantitative analysis to be conducted to explore the most effective ways to engage and treat this population within IAPT.

Finally, greater consideration should be given to involving partners/close family members, both as a resource of support for mothers, and also in order for therapists to gain insight and provide support for the difficulties they may be facing. This is especially vital given the importance of assessing for domestic violence, which affects pregnancy, maternal and child outcomes (Howard et al., 2013).

\section{Acknowledgements}

The study team acknowledges the study delivery support given by the local Clinical Research Networks. The study team would also like to thank the women and therapists who gave up their time to take part in the study.

Ethical statement. The authors assert that all procedures contributing to this work comply with the ethical standards of the English National Health Service. Ethical approval was obtained from the Camberwell St Giles London Research Ethics Committee (REC reference: 13/LO/1855).

Conflicts of interest. The authors have no conflicts of interest with respect to this publication.

Financial support. This paper summarizes independent research funded by the National Institute for Health Research (NIHR) under its PGfAR Programme (grant reference number RP-PG-1210-12002). The views expressed are those of the authors and not necessarily those of the NHS, the NIHR or the Department of Health.

\section{References}

Bauer, A., Parsonage, M., Knapp, M., Iemmi, V. and Adelaja, B. (2014). Costs of perinatal mental health problems. London: Centre for Mental Health. Available at: http://eprints.lse.ac.uk/59885/ 
Braun, V. and Clarke, V. (2006). Using thematic analysis in psychology. Qualitative Research in Psychology, 3, 77-101. doi: 10.1191/1478088706qp063oa

Clark, D. M. (2011). Implementing NICE guidelines for the psychological treatment of depression and anxiety disorders: the IAPT experience. International Review of Psychiatry, 23, 318-327. doi: 10.3109/09540261.2011.606803

Cuijpers, P., Brännmark, J. G. and van Straten, A. (2008). Psychological treatment of postpartum depression: a meta-analysis. Journal of Clinical Psychology, 64, 103-118. doi: 10.1002/jclp. 20432

Cuijpers, P., van Straten, A., Bohlmeijer, E., Hollon, S. D. and Andersson, G. (2010). The effects of psychotherapy for adult depression are overestimated: a meta-analysis of study quality and effect size. Psychological Medicine, 40, 211-223. doi: 10.1017/S0033291709006114

Dagher, R. K., McGovern, P. M., Dowd, B. E. and Gjerdingen, D. K. (2012). Postpartum depression and health services expenditures among employed women. Journal of Occupational and Environmental Medicine, 54, 210-215. doi: 10.1097/JOM.0b013e31823fdf85

Department of Health (2013). IAPT Perinatal Positive Practice Guide. London. Available at: https://www.uea.ac.uk/documents/246046/11919343/perinatal-positive-practice-guide-2013.pdf/ aa054d07-2e0d-4942-a21f-38fba2cbcceb

Gavin, N. I., Meltzer-Brody, S., Glover, V. and Gaynes, B. N. (2015). Is population-based identification of perinatal depression and anxiety desirable? Identifying Perinatal Depression and Anxiety: Evidence-Based Practice in Screening, Psychosocial Assessment and Management, 11-31. doi: 10.1002/9781118509722.ch1

Guest, G., Bunce, A. and Johnson, L. (2006). How many interviews are enough? An experiment with data saturation and variability. Field Methods, 18, 59-82. doi: 10.1177/1525822X05279903

Hamilton, S., Hicks, A., Sayers, R., Faulkner, A., Larsen, J., Patterson, S. and Pinfold, V. (2011). A user-focused evaluation of IAPT services in London. Report for Commissioning Support for London. Available at: https://www.researchgate.net/profile/John_Larsen/publication/279694132_A_ user-focused_evaluation_of_IAPT_services_in_London/links/5597bbc008ae21086d222659.pdf.

Health and Social Care Information Centre (2014). Psychological Therapies: Annual Report on the use of IAPT services: England 2014/15. Available at: http://content.digital.nhs.uk/catalogue/PUB19098/ psyc-ther-ann-rep-2014-15.pdf

Health Education England (2017). Perinatal mental health: England 2017. Available at: https://hee.nhs.uk/our-work/hospitals-primary-community-care/mental-health-learning-disability/ mental-health/perinatal-mental-health

Howard, L. M., Oram, S., Galley, H., Trevillion, K. and Feder, G. (2013). Domestic violence and perinatal mental disorders: a systematic review and meta-analysis. PLoS Medicine, 10, e1001452.

Howard, L. M., Molyneaux, E., Dennis, C. L., Rochat, T., Stein, A. and Milgrom, J. (2014). Nonpsychotic mental disorders in the perinatal period. The Lancet, 384, 1775-1788. doi: 10.1016/S01406736(14)61276-9

Joint Commissioning Panel for Mental Health (2012). Guidance for commissioners of primary mental health care services. London: Royal College of Psychiatrists. Available at: https://www.rcpsych.ac. uk/pdf/perinatal_web.pdf

Khan, L. (2015). Falling through the gaps: perinatal mental health and general practice. London: Centre for Mental Health. Available at: http://www.rcgp.org.uk/ / /media/Files/CIRC/ Perinatal-Mental-Health/RCGP-Exec-Summary-Falling-through-the-gaps-PMH-and-generalpractice-Mar-2015.ashx

Megnin-Viggars, O., Symington, I., Howard, L. M. and Pilling, S. (2015). Experience of care for mental health problems in the antenatal or postnatal period for women in the UK: a systematic review and meta-synthesis of qualitative research. Archives of Women's Mental Health, 18, 745-759. doi: 10.1007\%2Fs00737-015-0548-6 
O’Mahen, H., Fedock, G., Henshaw, E., Himle, J. A., Forman, J. and Flynn, H. A. (2012). Modifying CBT for perinatal depression: what do women want?: a qualitative study. Cognitive and Behavioral Practice, 19, 359-371. doi: 10.1016/j.cbpra.2011.05.005

O'Mahen, H., Richards, D.A., Woodford, J., Wilkinson, E., McGinley, J., Taylor, R.S. et al. (2014). Netmums: a phase II randomized controlled trial of a guided Internet behavioural activation treatment for postpartum depression. Psychological Medicine, 44, 1675-1689. doi: 10.1017/S0033291713002092

Richards, D. A., Weaver, A., Utley, M., Bower, P., Cape, J., Gallivan, S. et al. (2010). Developing evidence-based and acceptable stepped care systems in mental health care: an operational research project. Final Report, NIHR Service Delivery and Organisation Programme. Available at: http://www. netscc.ac.uk/hsdr/files/project/SDO_ES_08-1504-109_V01.pdf

Stein, A., Pearson, R. M., Goodman, S. H., Rapa, E., Rahman, A., McCallum, M. and Pariante, C. M. (2014). Effects of perinatal mental disorders on the fetus and child. The Lancet, 384, 1800-1819. doi: 10.1016/S0140-6736(14)61277-0 